\title{
Detección de genes Epl1 y Sm1 en Trichoderma spp. antagonistas contra hongos fitopatógenos
}

\author{
Detection of Epll and Sml genes in Trichoderma spp. antagonists against \\ phytopatogenic fungi
}

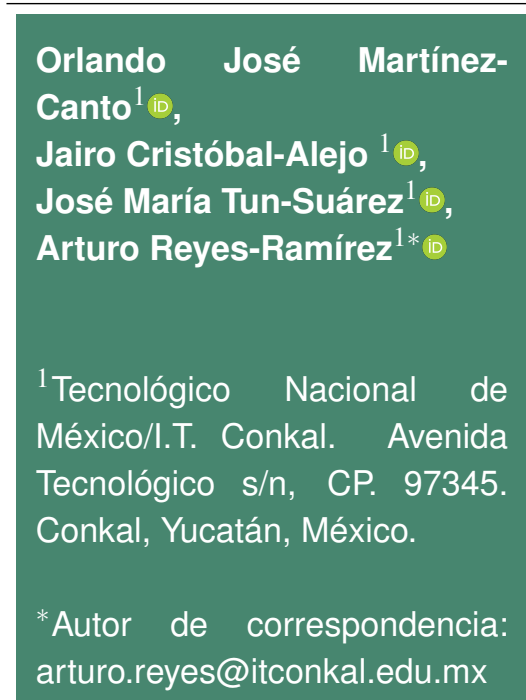

Nota científica

Recibida: 09 de enero 2021

Aceptada: 03 de agosto 2021

Como citar: Martínez-Canto OJ, Cristóbal-Alejo J, TunSuárez JM, Reyes-Ramírez A (2021) Detección de genes Epl1 y Sm1 en Trichoderma spp. antagonistas contra hongos fitopatógenos. Ecosistemas y Recursos Agropecuarios 8(2): e2791. DOI: 10.19136/era.a8n2.2791
RESUMEN. Trichoderma spp. se utilizan como método de biocontrol de fitopatógenos causantes de pérdidas agrícolas. El objetivo de este trabajo fue identificar nueve cepas de Trichoderma, determinar la capacidad antagónica contra Fusarium oxysporum y Corynespora cassiicola, así como los genes que codifican los elicitores $S m 1$ y Ep/1 de la resistencia sistémica en plantas. La capacidad antagónica contra fitopatógenos se determinó mediante enfrentamientos duales. La identificación molecular correspondió a $T$. asperellum (19-31), T. erinaceum (10-15), T. ghanense (21-39) y $T$. harzianum (18-31, 05E-60, 20-35, 13-01, 01-01, 29-54). Las cepas, 10-15, 19-31 y 05E-60 presentaron mayor capacidad de inhibición micelial contra F. oxysporum, mientras las cepas $32-09$ y 27-08 contra $C$. cassiicola. Los metabolitos volátiles de las cepas 27-08, 21-39 y 05E-60 inhibieron el crecimiento de F. oxysporum. La presencia de los genes Ep/1 fueron identificados en las cepas 19-31 y 10-15, y $S m 1$ en las cepas 27-08 y 32-09. Palabras clave: Corynespora cassiicola, Fusarium oxysporum, inhibición micelial, metabolitos volátiles.

ABSTRACT. Trichoderma spp. are used as a method of biocontrol of phytopathogenic that cause agricultural losses. The objective of this work was to identify nine Trichoderma strains, determine the antagonistic capacity against Fusarium oxysporum and Corynespora cassiicola, as well as the genes that encode the elicitors Sm1 and Ep/1 of systemic resistance in plants. The antagonistic capacity against phytopathogens was determined through dual confrontations. The molecular identification corresponded to T. asperellum (19-31), T. erinaceum (10-15), T. ghanense (21-39) and $T$. harzianum (18-31, 05E-60, 20-35, 13-01, 01-01, 29-54). Strains 10-15, 19-31 and 05E-60 showed greater mycelial inhibition capacity against $F$. oxysporum, while the strains 32-09 and 27-08 against $C$. cassiicola. The volatile metabolites of 27-08, 21-39 and 05E-60 strains inhibited the growth of $F$. oxysporum. The presence of the Ep/1 genes were identified in 19-31 and 10-15 strains, and $S m 1$ in 27-08 and 32-09 strains.

Key words: Corynespora cassiicola, Fusarium oxysporum, micelial inhibition, volatile metabolites. 


\section{INTRODUCCIÓN}

En las últimas décadas el manejo para el control de plagas y enfermedades que afectan a los cultivos ha sido mediante el uso excesivo de plaguicidas que tienen un severo impacto negativo sobre la salud humana y el medio ambiente (Adnan et al. 2019). Una alternativa efectiva para combatir los patógenos presentes en el suelo y mitigar los efectos adversos que ocasionan los compuestos organosintéticos es el empleo de microorganismos como biocontroladores (Al-askar et al. 2021, Ghoniem et al. 2021). Al respecto, se sabe que Trichoderma, organismo simbionte de plantas de bajo costo, efectivo y amigable con el ambiente (Sood et al. 2020) ejerce mecanismos de control por micoparasitismo, competencia, antibiosis, suprime la actividad de organismos patógenos por actividades enzimáticas, mantiene la micro flora del suelo y aumenta el crecimiento y producción de las plantas (Thapa et al. 2020). Además de que induce resistencia y tolerancia contra estrés biótico y abiótico (Alfiky y Weiskopff 2021).

Con el tiempo las plantas han desarrollado mecanismos de defensa, la presencia de patógenos es capaz de activar, mediante señales, múltiples respuestas protectoras tanto locales como sistémicas, que logran disminuir la posibilidad de infecciones y reducir el impacto de una plaga (Enebe y Babalola 2019). Se sabe que Trichoderma posee la facultad de activar el sistema inmune de las plantas (Aamir et al. 2019). La resistencia sistémica inducida (RSI), generalmente señalada como un método o un fenomeno para mejorar el sistema inmunológico inherente de la planta, también la resistencia sistémica adquirida (RSA), importante en la mejora de defensa de las plantas contra patógenos, que se refiere a una vía de transducción de señales (Murali et al. 2021). Estos mecanismos de defensa son regulados por diferentes tipos de elicitores, como las proteínas Sm1 y su homóloga, Epl1 (Tanaka y Kahmann 2021). Tanto Sm1 como Ep/1 son proteínas no catalíticas que pertenecen a la familia de las cerato-plataninas, bastante comunes en microorganismos en los que realizan diferentes funciones (Ramírez-Valdespino et al. 2019), en organismos patógenos son indispensables para lograr una patogenicidad, y también necesarias e importantes en la señalización de las moléculas que intervienen en la inducción de resistencia en las plantas (Chen et al. 2020). La proteína Sm1 activa la resistencia sistémica inducida en las primeras etapas de la interacción planta-Trichoderma mediante la acumulación del ácido jasmónico (AJ) y etileno (ET) (Guzmán-Guzmán et al. 2019), impulsada por la acción de los organismos necrotróficos, otorgándole a la planta mayor capacidad de respuesta frente a infecciones, lo que resulta en una estrategia de defensa efectiva (Salas-Marina et al. 2015). Mientras que la proteína Ep/1 producida de forma endógena en la planta y esencial para la señalización de las vías del ácido salicílico (AS), induce la resistencia sistémica adquirida como medida de defensa al ataque de patógenos biotróficos y hemibiotróficos, y se dispersa en toda la planta (Gomes et al. 2017). Si bien, las vías de señalización del AS, AJ y ET para inducir la resistencia en las plantas son mutuamente antagonistas, se ha evidenciado que entre algunas especies de Trichoderma hay correlación entre estas fitohormonas debido a la sobreposición de genes relacionados a la RSI y RSA detectados en plantas tratadas con Epl1 y Sm1 (Cheng et al. 2018), lo que sugiere que ambos elicitores funcionan de forma sinérgica en los dos sistemas de defensa inducido por Trichoderma (Salas-Marina et al. 2015). Con base en lo anterior el objetivo del presente trabajo fue identificar, por medio de biología molecular, cepas de Trichoderma spp.; además de evaluar, in vitro, la eficacia contra F. oxysporum y C. cassiicola, así como determinar los genes que codifican los elicitores Sm1 y Ep/1 relacionados con la resistencia sistémica en las plantas.

\section{MATERIALES Y MÉTODOS}

\section{Microorganismos utilizados}

En total se utilizaron 13 cepas de Trichoderma spp. nueve cepas sin identificar, una identificada molecularmente ( $T$. asperellum Ta13-17, GenBank: MH015346.1), y tres cepas reportadas, $T$. virens 27-08, T. virens 32-09 (Moo et al. 2018) 
y T. virens 05-02 (Candelero et al. 2015), todas de la colección del laboratorio de Fitopatología del TecNM/Campus Conkal. Los fitopatógenos F. oxysporum y $C$. cassiicola fueron aislados previamente de Capsicum chinense.

\section{Identificación molecular de Trichoderma spp}

Para la identificación a nivel de especie, se realizaron extracciones de DNA a partir de cultivos monospóricos en medio agar dextrosa y papa (PDA) (BD Bioxon ${ }^{\circledR}$, México) de cinco días de crecimiento a $28^{\circ} \mathrm{C}$, se realizó un raspado superficial del micelio de las placas Petri y se utilizó el Kit ZR Fungal/Bacterial DNA MiniPrep ${ }^{T M}$ (Zymo Research Corp, USA). La identificación se realizó mediante las secuencias internas ITS1-5.8S-ITS2 del ARN ribosomal (White et al. 1990). Los productos de PCR se enviaron a secuenciar a la empresa Psomagen USA. Las secuencias obtenidas se analizaron en el programa BioEdit Sequence Alignment Editor y los resultados se compararon con la base de datos del National Center for Biotechnology Information (NCBI), mediante el programa Basic Local Alignment Search Tool (BLAST).

\section{Antagonismo de Trichoderma spp. contra F. oxys- porum y C. cassiicola}

Para evaluar el antagonismo de Trichoderma spp. contra $F$. oxysporum y C. cassiicola se utilizó la técnica de cultivos duales, para lo cual se utilizaron cajas de Petri con medio PDA y se sembraron diametralmente en dirección opuesta un disco de micelio activo de los fitopatógenos y de las cepas de Trichoderma. Las cajas se incubaron a $28^{\circ} \mathrm{C}$, y se realizaron mediciones del crecimiento micelial cada $24 \mathrm{~h}$. Para la prueba de metabolitos volátiles se utilizaron dos cajas de Petri de $70 \mathrm{~mm}$ con medio PDA, una sobrepuesta encima de otra, de tal manera que en el centro de la caja colocada como base (caja inferior) se sembró un disco de micelio de cada cepa de Trichoderma, mientras que en la tapa (caja superior) un disco de micelio de los fitopatógenos, y se incluyó como testigo el patógeno sin el antagonista. Las cajas se sellaron con doble capa de parafilm, se mantuvieron a temperatura constante de $28^{\circ} \mathrm{C}$ y se midió el crecimiento del micelio cada $24 \mathrm{~h}$ (Dennis y
Webster 1971). La medición se realizó hasta observar que alguna cepa cubriera por completo el área total de la caja y se comparó con el control en ausencia del antagonista. El efecto antagónico de cada cepa se calculó mediante la medición de la inhibición del crecimiento micelial.

\section{Determinación de genes Sm1 y Epl1}

El diseño de iniciadores específicos Sm1 y Epl1, se realizó por medio de una búsqueda de las secuencias de cada gen mediante el programa BLAST del NCBI. Se obtuvieron los marcadores de secuencia expresada (EST) de cada gen, a partir de los cuales se diseñaron los iniciadores mediante edición manual y con el programa Pimer3 Input (versión 0.4.0) (Untergasser et al. 2007). Se diseñaron dos pares iniciadores para cada gen, para $S m 1$ se utilizaron dos secuencias del gen que codifica para una proteína pequeña de $T$. virens (DQ121133 y XM_014104331) (iniciador directo 5'-TCTCCTACGACACCGGCTAC-3' y reverso 5'-GAGACCGCAGTTCTTAACAG-3'), con un tamaño de 353 bp. Para el gen Epl1 se utilizaron las secuencias de T. atroviride (AJ9018799) y de T. asperellum (AJ902344); (iniciador directo 5'-CTTCAAGCTTGCTCTCTTCA-3') y reverso (5'GCCAAGACGTAGATGGTCTT- 3') con un tamaño de 267 bp. Las reacciones por PCR se realizaron bajo las siguientes condiciones: desnaturalización inicial a $94{ }^{\circ} \mathrm{C}$ durante $3 \mathrm{~min} ; 30$ ciclos de desnaturalización de $94{ }^{\circ} \mathrm{C}$ por $1 \mathrm{~min}$, alineamiento a $53{ }^{\circ} \mathrm{C}$ por 30 s y extensión durante $45 \mathrm{~s}$ a $72{ }^{\circ} \mathrm{C}$; con extensión final de $72{ }^{\circ} \mathrm{C}$ durante $5 \mathrm{~min}$, y almacenamiento final a $10^{\circ} \mathrm{C}$. Los productos de PCR se visualizaron por medio de electroforesis en gel de agarosa al $2 \%$ (Sigma, USA) y se tiñeron con SYBR ${ }^{T M}$ Green (Invitrogen, USA). Los productos de PCR se enviaron a secuenciar a la empresa Psomagen USA. Las secuencias obtenidas se analizaron en el programa BioEdit Sequence Alignment Editor y, posteriormente, los consensos se obtuvieron mediante el programa EMBOSS Needle Pairwise Sequence Alignment, y se compararon los resultados con la base de datos del NCBI mediante el programa BLAST. 


\section{Análisis estadístico de datos}

En la evaluación de Trichoderma como antagonista de $F$. oxysporum y C. cassiicola cada prueba se realizó por triplicado. Con los datos que arrojaron las variables de respuesta se realizó un análisis de varianza y se utilizó el método Tukey ( $p$ $\leq 0.05)$ para comparar las medias, con la ayuda del programa InfoStat versión 2020.

\section{RESULTADOS Y DISCUSIÓN}

Con base a las características morfológicas y las secuencias internas ITS1-5.8S-ITS2 del ARN ribosomal, la identificación de los aislados corresponde a T. asperellum, T. erinaceum, T. ghanense y seis aislados de $T$. harzianum (Tabla 1). La variedad entre especies de Trichoderma ha sido reportada, siendo común aislar $T$. harzianum, la diversidad intra e interespecífica es generada por las diferentes condiciones en donde se ha desarrollado los aislados de Trichoderma, llegando a tener diferencias en sus características (Moo et al. 2018).

De las 13 cepas de Trichoderma evaluadas contra la inhibición del crecimiento micelial de F. oxysporum y $C$. casiicola, $T$. erinaceum 10-15 fue la que mostró mayor inhibición contra F. oxysporum, con un $90.5 \%$, seguido de las cepas T. asperellum 19-31 con $84.9 \%$, y $T$. harzianum $05 \mathrm{E}-60$ con $84.5 \%$. Al respecto, Aamir et al. (2019) evaluaron la capacidad antagónica in vitro de diversas especies de Trichoderma contra F. oxysporum f. sp. lycopersici y reportaron que $T$. erinaceum registró la mayor capacidad inhibitoria micelial de F. oxysporum, seguido de $T$. asperellum y $T$. longibranchiatum, resultados que concuerdan con los de este estudio. Por otro lado, estudios previos indican que $T$. asperellum tiene una capacidad de inhibición micelial de hasta $81 \%$ para $F$. incarnatum e inhibición total de F. oxysporum al evaluar diferentes concentraciones de ácido cítrico obtenido de esta especie (Al-Askar et al. 2021, Intana et al. 2021). En tanto que $C$. cassiicola, en la inhibición micelial T. virens 32-09 tuvo una capacidad de inhibición de $75.1 \%$, seguido de la cepa $T$. virens $05-02$ con $71.6 \%$ (Tabla 2); trabajos con especies de Trichoderma han demostrado la capacidad de inhibir el crecimiento de C. cassiicola, en particular con $T$. asperellum y T. harzianum (Maza et al. 2012). Sobre lo mismo, Baiyee et al. (2019) reportan que T. spirale T76-1 inhibió el crecimiento micelial de $C$. cassiicola y Curvularia aeria, en un 84.68 y $93.03 \%$, respectivamente, los cuales son hongos causantes de la mancha foliar en lechuga (Lactuca sativa). Esto podría sugerir que las cepas de Trichoderma secretan más, o producen metabolitos más fuertes que inhiben el crecimiento micelial de fitopatógenos (Li et al. 2019), además de que las especies de Trichoderma fueron aislados de diferentes lugares, lo que puede interpretarse como un ejemplo de que las especies pueden desarrollarse mediante una selección forzada por el ambiente o las condiciones ecológicas particulares (Moo et al. 2018). Trichoderma ha demostrado ser un hongo muy flexible, tiene una alta capacidad reproductiva, es muy eficiente en la toma y utilización de nutrientes, además de ser muy agresivo contra hongos fitopatógenos (Contreras-Cornejo et al. 2016, Adnan et al. 2019).

En lo que se refiere a metabolitos volátiles, las 13 cepas de Trichoderma evaluadas contra F. oxysporum y $C$. cassiicola mostraron efecto inhibitorio sobre el crecimiento de los hongos fitopatógenos, en relación al testigo. Sin embargo, T. harzianum 05E-60 tuvo mayor inhibición en el crecimiento micelial sobre C. Cassiicola con $71.1 \%$, seguido de T. erinaceum 10 15 con $58.5 \%$ (Tukey, $p \leq 0.05$ ) (Tabla 2). Por otro lado, en relación al efecto inhibitorio de crecimiento de F. oxysporum, T. virens 27-08 tuvo una mayor inhibición micelial con $46.3 \%$, seguido de $T$. ghanense $21-39$ con $39.7 \%$ y $T$. harzianum $05 \mathrm{E}-60$ con $38 \%$. Se conoce que Trichoderma spp. tienen la capacidad de producir metabolitos que generan fungistasis, es decir, inhiben la germinación de esporas de sus competidores, lo que le otorga ventaja al competir por espacio y nutrientes (El-Sharkawy et al. 2018). Al respecto, Osorio et al. (2016) reportaron resultados similares al evaluar la inhibición de Trichoderma sp. sobre el crecimiento de Rhizoctonia solani con un efecto inhibitorio de entre 7.81 y $51.56 \%$. Del mismo modo, Baiyee et al. (2019) evaluaron la capacidad inhibitoria de $T$. espirale T76-1 sobre $C$. cassiicola y Curvularia aeria y reportaron una inhibición del 41.29 
Tabla 1. Identificación molecular de cepas de Trichoderma spp.

\begin{tabular}{|c|c|c|c|c|}
\hline \multirow[b]{2}{*}{ Aislado } & \multirow[b]{2}{*}{ Localidad } & \multicolumn{3}{|c|}{ Homología con el NCBI-GenBank } \\
\hline & & Especie & Identidad (\%) & No. accesión \\
\hline $19-31$ & Tzucacab $^{1}$ & T. asperellum & 99.83 & MH911417.1 \\
\hline $10-15$ & Buctzotz $^{2}$ & T. erinaceum & 100 & MH748164.1 \\
\hline $21-39$ & Peto $^{1}$ & T. ghanense & 100 & MF078652.1 \\
\hline $18-31$ & Halachó $^{2}$ & T. harzianum & 99.67 & KF201295.1 \\
\hline $05 \mathrm{E}-60$ & Dzilam González ${ }^{2}$ & T. harzianum & 99.17 & KJ767088.1 \\
\hline $20-35$ & Tzucacab $^{2}$ & T. harzianum & 99.84 & KF201295.1 \\
\hline 13-01 & Conkal $^{2}$ & T. harzianum & 100 & MH153633.1 \\
\hline 01-01 & Tizimín ${ }^{1}$ & T. harzianum & 100 & MH153633.1 \\
\hline $29-54$ & Akil $^{1}$ & T. harzianum & 100 & MH153633.1 \\
\hline
\end{tabular}

Tabla 2. Inhibición del crecimiento micelial y por metabolitos volátiles de Trichoderma spp. contra hongos fitopatógenos.

\begin{tabular}{lllll}
\hline & \multicolumn{2}{c}{ Inhibición del crecimiento micelial (\%) } & \multicolumn{2}{c}{ Inhibición por volátiles $(\%)$} \\
\cline { 2 - 5 } Especie & F. oxysporum & C. cassiicola & F. oxysporum & C. cassiicola \\
\hline T. asperellum 19-31 & $84.9 \pm 1.5^{a b}$ & $32.1 \pm 18.1^{\text {cde }}$ & $15.9 \pm 7.5^{b c}$ & $33.7 \pm 3.4^{d e}$ \\
T. erinaceum 10-15 & $90.5 \pm 2.4^{a}$ & $37.8 \pm 5.9^{b c d e}$ & $14.3 \pm 4.0^{b c}$ & $58.5 \pm 6.4^{a b}$ \\
T. ghanense 21-39 & $81.1 \pm 2.3^{b}$ & $34.2 \pm 2.5^{c d e}$ & $39.7 \pm 4.9^{a}$ & $45.0 \pm 7.4^{b c d e}$ \\
T. harzianum 18-31 & $83.3 \pm 5.1^{a b}$ & $24.7 \pm 11.1^{c d e}$ & $31.5 \pm 6.6^{a b}$ & $43.8 \pm 2.3^{b c d e}$ \\
T. harzianum 05E-60 & $84.5 \pm 4.9^{a b}$ & $46.0 \pm 5.5^{b c d e}$ & $38.0 \pm 5.9^{a}$ & $71.1 \pm 6.2^{a}$ \\
T. harzianum 13-01 & $80.9 \pm 1.7^{b c}$ & $50.9 \pm 2.9^{a b c d}$ & $27.4 \pm 4.6^{a b}$ & $56.0 \pm 6.4^{a b c}$ \\
T. harzianum 01-01 & $83.7 \pm 0.4^{a b}$ & $22.0 \pm 12.6^{e}$ & $27.8 \pm 2.0^{a b}$ & $47.3 \pm 9.0^{b c d}$ \\
T. harzianum 20-35 & $80.3 \pm 4.1^{b c}$ & $24.1 \pm 7.6^{d e}$ & $35.6 \pm 1.7^{a b}$ & $51.0 \pm 5.7^{b c d}$ \\
T. harzianum 29-54 & $84.1 \pm 1.7^{a b}$ & $34.9 \pm 7.5^{c d e}$ & $6.1 \pm 2.5^{c d}$ & $56.2 \pm 9.2^{a b c}$ \\
T. asperellum 13-17 & $80.1 \pm 5.9^{b c}$ & $52.0 \pm 8.0^{a b c}$ & $4.3 \pm 4.0^{c d}$ & $51.4 \pm 5.8^{a b c d}$ \\
T. virens 27-08 & $59.6 \pm 8.4^{e}$ & $65.5 \pm 3.5^{a b}$ & $46.3 \pm 6.6^{a}$ & $26.3 \pm 7.8^{e}$ \\
T. virens 32-09 & $68.4 \pm 4.9^{e d}$ & $75.1 \pm 7.9^{a}$ & $24.1 \pm 4.1^{a b}$ & $36.3 \pm 6.1^{c d e}$ \\
T. virens 05-02 & $73.7 \pm 2.4^{c d}$ & $71.6 \pm 2.3^{a}$ & $33.2 \pm 6.5^{a b}$ & $46.2 \pm 9.9^{b c d}$ \\
Testigo & $0.0 \pm 0.0^{f}$ & $0.0 \pm 0.0^{f}$ & $0.0 \pm 0.0^{d}$ & $0.0 \pm 0.0^{f}$ \\
\hline Medias con letra diferente en la misma columna son estadísticamente diferentes (Tukey, p $\leq 0.05)$
\end{tabular}

y $42.35 \%$, respectivamente, señalando que los compuestos volátiles, junto con la competición y las actividades enzimáticas, son mecanismos involucrados en el biocontrol. Esta capacidad de producir metabolitos secundarios le otorga a Trichoderma spp. ventajas selectivas en procesos como competencia, simbiosis, transporte de metales, señalización y actividad micoparasítica (Manganiello et al. 2018).

Para comprobar la presencia los genes Ep/1 y Sm1 que codifican proteínas reportadas como promotoras de la RSI en plantas, se realizó el análisis de las secuencias de nucleótidos obtenidos de los fragmentos de PCR, donde las secuencias de $T$. asperellum 19-31 y T. erinaceum 10-15 mostraron homología con el gen Epl1; mientras que T. virens 2708 y T. virens 32-09 con el gen Sm1 (Tabla 3). Esto revela que los genes Sm1 y Epl1 tienen regiones con- servadas entre las secuencias de un mismo grupo de hongos previamente reportados. Al respecto, se sabe que estos genes del género Trichoderma codifican proteínas que realizan diversas funciones en su interacción con las plantas. $S m 1$ se ha reportado principalmente en $T$. virens, lo que concuerda con los resultados hallados en este trabajo, cuya función en las plantas es la inducción de genes relacionados con la defensa, la producción de especies reactivas de oxígeno y compuestos fenólicos (Ramírez-Valdespino et al. 2019); sin embargo, Freitas et al. (2013) evidenciaron la presencia de $S m 1$ en $T$. atroviride y $T$. harzianum, pero sin determinar su función. Por otro lado, se ha reportado que Ep/1, proteína elicitora de la inducción de genes relacionados con la defensa de la planta y de la activación del sistema inmune (Ramírez-Valdespino et al. 2019), está presente en 
Tabla 3. Identificación de genes involucrados en la Resistencia Sistémica en Trichoderma spp.

\begin{tabular}{lcccc}
\hline & \multicolumn{4}{c}{ Homología en el GenBank } \\
\cline { 2 - 5 } Especie & Gen & Identidad (\%) & e-valor & Acces. GenBank \\
\hline T. asperellum 19-31 & Ep/1 & 100.00 & $2 \mathrm{e}-163$ & JN966997 \\
T. erinaceum 10-15 & Ep/1 & 98.62 & $7 \mathrm{e}-103$ & XM_014082295 \\
T. virens 27-08 & Sm1 & 97.70 & $5 \mathrm{e}-169$ & DQ494198 \\
T. virens 32-09 & Sm1 & 99.12 & $1 \mathrm{e}-170$ & DQ121133 \\
\hline
\end{tabular}

un mayor número de especies de Trichoderma, en el presente trabajo se detectó en la especie $T$. erinaceum y, en la especie $T$. asperellum resultado similar a lo reportado por Yu et al. (2018), además de que estudios previos lo reportan en $T$. longibrachiatum (Vargas et al. 2008), T. atroviridae (Salas-Marina et al. 2015), T. harzianum (Gomes et al. 2017) y T. formosa (Cheng et al. 2018). Tanto Sm1, como su ortóloga Ep/1 son moléculas señalizadoras esenciales para lograr la aceleración en la resistencia, local y sistémica, de las plantas. De manera general, Sm1 interviene en la inducción de la RSI que se basa en la señalización del AJ y ET, mientras que Ep/1 participa en la señalización del AS, necesario para la RSA (Gomes et al. 2017, Ramírez-Valdespino et al. 2019).

Las especies $T$. erinaceum 10-15, $T$. asperellum 19-31 y $T$. harzianum 05E-60 inhibieron en mayor porcentaje el crecimiento micelial de $F$. oxysporum; mientras que $T$. virens 32-09 y $T$. virens 05-02 tuvieron los mejores porcentajes de inhibición contra $C$. cassiicola. Los metabolitos volátiles de $T$. virens 27-08, T. ghanense 21-39 y T. harzianum 05E60 mostraron mayor efecto inhibitorio contra $F$. oxysporum, pero solo $T$. harzianum $05 \mathrm{E}-60$ destacó en la inhibición de crecimiento micelial contra $C$. cassiicola. Cuatro cepas presentaron genes que codifican para proteínas relacionadas con la resistencia sistémica; en $T$. asperellum 19-31 y $T$. erinaceum 10-15 se identificó a Epl1, mientras que en T. virens 27-08 y $T$. virens 32-09 a Sm1. Por lo que estas cepas son una opción para el control de fitopatógenos como Fusarium oxysporum y Corynespora cassiicola y con el potencial de activar la respuesta sistémica en las plantas.

\section{LITERATURA CITADA}

Aamir M, Kashyap SP, Zehra A, Dubey MK, Singh VK, Ansari WA, Upadhyay RS, Singh S (2019) Trichoderma erinaceum bio-priming modulates the WRKYs defense programming in tomato against the Fusarium oxysporum f. sp. lycopersicy (Fol) challenged condition. Frontiers in Plant Science 10: 911. DOI: 10.3389/fpls.2019.00911.

Adnan M, Islam W, Shabir A, Khan KA, Ghramh HA, Huang Zhiquun Chen HY, Lu G (2019) Plant defense against fungal pathogens by antagonistic fungi with Trichoderma in focus. Microbial Pathogenesis 129: 7.18. DOI: 10.1016/j.micpath.2019.01.042.

Al-Askar AA, Saber WI, Ghoneem KM, Hafez EE, Ibrahim AA (2021) Crude citric acid of Trichoderma asperellum: tomato growth promotor and suppressor of Fusarium oxysporum f. sp. lycopersicy. Plants 10: 222. DOI: 10.3390/plants10020222.

Alfiky A, Weisskopf $L$ (2021) Deciphering Trichoderma-plant-pathogen interactions for better development of biocontrol applications. Journal of Fungi 7: 1-18. DOI: 10.3390/jof7010061.

Baiyee B, Pornsuriya C, Ito S, Sunpapao A (2019) Trichoderma spirale T76-1 displays biocontrol activity against leaf spot on lettuce (Lactuca sativa L.) caused by Corynespora cassiicola or Curvularia aeria. Biological Control 129: 125-200. 
Chen J, Karuppiah V, Dou D (2020) Multiplayer interaction of Trichoderma and plant in the induced plant resistance. In: Gupta VK, Zeilinger S, Singh HB, Druzhinina I (eds). New and future developments in microbial biotechnology and bioengineering: Recent developments in Trichoderma research. Elsevier. Amsterdam, Netherlands. pp: 141-155.

Cheng CH, Shen BN, Shang QW, Liu LYD, Peng KC, Chen YH, Chen FF, Huu SF, Wang YT, Wang HC, Wu HY, Lo CT, Lin SS (2018) Gene-to-gene network analysis of the mediation of plant innate immunity by the eliciting plant response-like 1 (Ep/1) Elicitor of Trichoderma formosa. Molecular Plant-Microbe Interactions 31: 683-691.

Candelero DJ, Cristóbal AJ, Reyes R A, Gamboa AMM, Ruíz SE, Tun SJM (2015) Trichoderma spp. fostering growth on Capsicum chinense Jacq. seedlings and antagonistic against Meloidogyne incognita. Phyton, International Journal of Experimental Botany 84: 113-119.

Contreras-Cornejo HA, Macías-Rodríguez L, Del Val E, Larsen J (2016) Ecological functions of Trichoderma spp. and their secondary metabolites in the rhizosphere: interactions with plants. FEMS Microbiology Ecology 92: 1-17.

Dennis C, Webster J (1971) Antagonistic properties of species group of Trichoderma II production of non-volatile antibiotics. Transactions of the British Mycological Society 57: 41-48.

El-Sharkawy HH, Rashad YM, Ibrahim SA (2018) Biocontrol of stem rust disease of wheat using arbuscular mycorrhizal fungi and Trichoderma spp. Physiological and Molecular Plant Pathology 103: 84-91.

Enebe MC, Babalola OO (2019) The impact of microbes in the orchestration of plants resistance to biotic stress: a disease management approach. Applied Microbiology and Biotechnology 103: 9-25.

Freitas RS, Steindorff AS, Ramada MHS, De Siqueira SJL, Noronha EF, Ulhoa CJ (2013) Cloning and characterization of a protein elicitor Sm1 gene from Trichoderma harzianum. Biotechnology Letters 36: 783-788.

Gomes EV, Ulhoa CJ, Cardoza RE, Silva RN, Gutiérrez S (2017) Involvement of Trichoderma harzianum Epl-1 protein in the regulation of botrytis virulence- and tomato defense-related genes. Frontiers in Plant Science 8: 1-11. DOI: 10.3389/fpls.2017.00880.

Ghoniem AA, Abd E-Hai KM, El-khateeb AY, Eldadamony NM, Mahmoud SF, Elsayed A (2021) Enhancing the Potentiality of Trichoderma harzianum against Pythium pathogen of beans using chamomile (Matricaria chamomilla, L.) Flower Extract. Molecules 26: 1178. DOI: 10.3390/molecules26041178.

Guzmán-Guzmán P, Porras-Troncoso MD, Olmedo-Bonfil V, Herrera-Estrella A (2019) Trichoderma species: versatile plant symbionts. Phytopatology 52: 1-43. DOI: 10.1094/PHYTO-07-18-0218-RVW.

Intana W, Kheawleng S, Sunpapo A (2021) Trichoderma asperellum T76-14 released volatile organic compounds against postharvest fruit rot in muskmelons (Cucumis melo) caused by Fusarium incarnatum. Journal of Fungi 7: 46. DOI: 10.3390/jof7010046.

Li N, Islam MT Kang S (2019) Secreted metabolite-mediated interactions between rhizosphere bacteria and Trichoderma biocontrol agents. PLoS ONE 14(12): e0227228. DOI: 10.1371/journal.pone.0227228.

Manganiello G, Sacco A, Ercolano MR, Vinale F, Lanzuise S, Pascale A, Napolitano M, Lombardi N, Lorito M, Woo SL (2018) Modulation of tomato responses to Rhizoctonia solani by Trichoderma harzianum and its secondary metabolite harzianic acid. Frontiers in Microbiology 9: 1966. DOI: 10.3389/fmicb.2018.01966.

Maza M, Starzonelli EA, Yasem MG (2012) Evaluación in vitro de aislamientos nativos de Trichoderma como agentes de biocontrol y promoción del crecimiento inicial en soja. Revista Agrononómica del Noroeste Argentino 32: 55-62. 
Moo F, Cristóbal J, Reyes A, Tun J, Gamboa M, Islas-Flores I (2018) Incompatibilidad interespecífica de especies de Trichoderma contra Meloidogyne incognita en Solanum lycopersicum. Scentia Fungorum 47: 37-45.

Murali M, Naziya B, Ansari MA, Alomary MN, AlYahya S, Almatroudi A, Thriveni MC, Gowtham HG, Singh SB, Aiyaz M, Kalegowda N, Lakshmidevi N, Amruthesh KN (2021) Bioprospecting of rhizosphere-resident fungi: Their role and importance in sustainable agriculture. Journal of Fungi 7: 314. DOI: 10.3390/jof7040314.

Osorio E, Hernández F, Rodríguez R, Varela S, Estrada B, López J (2016) Actividad antagónica de Trichoderma spp. sobre Rhizoctonia solani in vitro. Investigación y Ciencia 67: 5-11.

Ramírez-Valdespino CA, Casas-Flores S, Olmedo-Monfil V (2019) Trichoderma as a model to study effector-like molecules. Frontiers in Microbiology 10: 1-14. DOI: 10.3389/fmicb.2019.01030.

Salas-Marina MA, Isordia-Jasso MI, Islas-Osuna MA, Delgado-Sánchez P, Jiménez-Bremont JF, RodríguezKessler M, Rosales-Saavedra MT, Herrera-Estrella A, Casas-Flores S (2015) The Epl1 and Sm1 proteins from Trichoderma atroviride and Trichoderma virens differentially modulate systemic disease resistance against different life style pathogens in Solanum lycopersicum. Frontiers in Plant Science 6: 1-13. DOI: 10.3389/fpls.2015.00077.

Sood M, Kapoor D, Kumar V, Sheteiwy MS, Ramakrishnan M, Landi M, Araniti F, Sharma A (2020) Trichoderma: The "Secrets" of a multitalented biocontrol agent. Plants 9: 762. DOI: 10.3390/plants9060762.

Tanaka S, Kahmann R (2021) Cell wall-associated effectors of plant-colonizing fungi. Mycologia 113: 247-260.

Thapa S, Rai N, Limbu AK, Joshi A (2020) Impact of Trichoderma sp. in agriculture: A review. Journal of Biology and Today's World 9: 227. DOI: 10.35248/2322-3308.20.09.225.

Untergasser A, Nijveen H, Rao X, Bisseling T, Geurts R, Leunissen JA (2007) Primer3Plus, an enhanced web interface to Primer3. Nucleic Acids Research 35: 71-77.

Vargas WA, Djonovic S, Sukno SA, Kenerley CM (2008) Dimerization controls the activity of fungal elicitors that trigger systemic resistance in plants. Journal of Biological Chemistry 283: 19804-19815.

White TJ, Bruns TD, Lee SB, Taylor JW (1990) Amplification and direct sequencing of fungal ribosomal RNA genes for phylogenetics. In: Innis MA, Gelfand DH, Sninsky JJ, White TJ (eds.), PCR protocols: A guide to methods and applications. Academic Press. San Diego. pp: 315-321.

Yu W, Mijiti G, Huang Y, Fan H, Wang Y, Liu Z (2018) Functional analysis of eliciting plant response protein Epl1-Tas from Trichoderma asperellum ACCC30536. Scientific Reports 8: 1-13. DOI: 10.1038/s41598-01826328-1. 